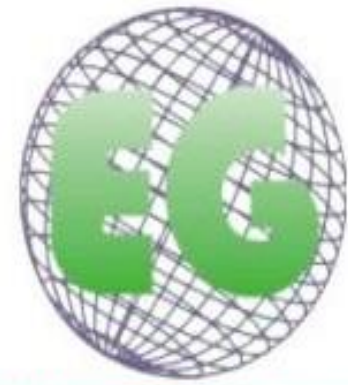

ISSN 1695-6141 $N^{\circ} 46$

\title{
¿Cómo ayuda la aplicación del proceso enfermero a las mujeres con diagnóstico ansiedad?
}

How can the application of the nursing process help to women with anxiety nursing diagnosis?

\section{*Amad Pastor, Miriam ${ }^{* *}$ Cánovas Tomás, Miguel Ángel ***Díaz García, Isidora}

* Enfermera Especialista en Salud Mental. Centro de Salud Mental Águillas. Servicio Murciano de Salud. E-mail: mirileda@hotmail.com **Enfermero. Hospital General Universitario Morales Meseguer. Servicio Murciano de Salud. *** Enfermera Especialista en Salud Mental. Centro de Salud Mental Lorca. Servicio Murciano de Salud. Murcia. España.

http://dx.doi.org/10.6018/eglobal.16.2.259881

\section{RESUMEN}

Objetivo: Evaluar las intervenciones grupales de enfermería en mujeres con diagnóstico enfermero ansiedad mediante el resultado NOC autocontrol de la ansiedad.

Material y método: Estudio pre-experimental del tipo pretest-postest de un solo grupo realizado en un Centro de Salud Mental de la Región de Murcia a un grupo de 12 mujeres en edad adulta que presentan el diagnóstico enfermero ansiedad. Se realizó a cada paciente la valoración de enfermería por Patrones Funcionales de Salud (PFS) antes y después de la asistencia a las 8 sesiones de intervenciones enfermeras grupales. Al comenzar y al finalizar el grupo, se cumplimentó el criterio de resultado NOC autocontrol de la ansiedad. Las intervenciones enfermeras fueron a) disminución de la ansiedad (5820), terapia de relajación simple (6040), educación sanitaria (5510), enseñanza grupo (5604) y grupo de apoyo (5430). Se tomó como variable independiente las intervenciones enfermeras y como variable dependiente las puntuaciones del resultado NOC, empleándose como instrumento de evaluación.

Resultados: El análisis del resultado NOC autocontrol de la ansiedad nos muestra diferencias significativas. Antes de las intervenciones, la media presenta el valor $22.5 \mathrm{y}$ tras las mismas de 37.8 con una desviación típica de 3.22 y 7.11 respectivamente. La mediana antes y después de las intervenciones corresponde con los valores 21 y 37.5 . El parámetro del test de los rangos de Wilcoxon corresponde con un valor de $\mathrm{Z}$ igual a -2.98 con una $\mathrm{p}$ igual a 0.003 .

Conclusiones: El estado, conducta y percepciones de las pacientes del grupo medido con el resultado autocontrol de la ansiedad, mejora tras recibir las intervenciones enfermeras. La puntuación global de los indicadores empleados en este resultado ha sido apropiada como medida de los efectos de las 
intervenciones.

Palabras clave: Clasificación de resultados de enfermería (NOC); intervenciones de enfermería; ansiedad; diagnóstico enfermero; proceso de enfermería.

\section{ABSTRACT}

Purpose: To evaluate nursing group interventions in women with anxiety nursing diagnosis by self NOC result of anxiety.

Methodology: Pre-experimental pretest-posttest study composed by a single group of twelve women in a Mental Health Center of the Region of Murcia. The participants were a group of women in adulthood presenting the nursing diagnosis anxiety. Each patient received 8 group nursing interventions. Before and after the group intervention, a nursing outcome criteria is filled for each patient. The following nursing interventions were used: Anxiety reduction (5820), simple relaxation therapy (6040), health education (5510), group education (5604) and support group (5430). The independent variable was nursing interventions and the dependent variable was NOC punctuations, being used as an assessment tool.

Results: The analysis of the NOC result anxiety self-control shows significant differences. Before the intervention, the mean was 22.5 and 37.8 after it with standard deviations of 3.22 and 7.11 for each one. The median was 21 before interventions and 37.5 after them. The Wilcoxon test shows a $Z$ value of 2.98 with $\mathrm{p} 0.003$.

Conclusion: The state, behaviour and perception of patients improve with nursing interventions received. The global punctuation of used clinical indicators is a good tool to measure nursing intervention effects.

Keywords: Nursing Outcomes Classification (NOC); nursing interventions; anxiety; nursing diagnosis; Nursing Process

\section{INTRODUCCIÓN}

Los trastornos de ansiedad forman el grupo de trastornos mentales más comunes en la población general ${ }^{(1,2)}$. La ansiedad y la angustia son síntomas de consulta muy frecuentes, la mayoría de las veces muy inespecíficos, reflejando insatisfacciones y frustraciones que se pueden enmascarar somáticamente ${ }^{(3)}$.

Ser hombre o mujer no sólo puede influir en la prevalencia de los trastornos mentales, sino también en la manifestación y expresión de los síntomas ${ }^{(4)}$. La mayor importancia de estas entidades en las mujeres está relacionada con el contexto sociocultural y las vivencias provocadas por factores psicosociales, por los consensos y los modelos estereotipados de roles para hombres y mujeres y los ideales de feminidad y masculinidad que imponen ${ }^{(5)}$. El informe sobre salud mental y género de la Organización Mundial de la Salud (OMS) en 2002 señala que las diferencias por sexo se dan precisamente en las alteraciones mentales comunes, entre ellas los trastornos de ansiedad, que se presentan con doble frecuencia en las mujeres ${ }^{(6)}$.

Los trastornos de ansiedad han sido objeto de estudio como enfermedades mentales en diferentes textos como el Manual Diagnóstico y Estadístico de los Trastornos Mentales V (DSM-V) y la Clasificación Internacional de Enfermedades en su décima edición $(\mathrm{CIE}-10)^{(7,8)}$. Si bien, en el campo de la enfermería, la cuestión de la ansiedad es recogida en la Taxonomía II de la NANDA-I como juicio clínico sobre la respuesta humana a problemas de salud o procesos vitales o la vulnerabilidad en la respuesta de un individuo, familia o comunidad. Así, el foco del diagnóstico ansiedad queda 
enmarcado en el dominio 9 afrontamiento/tolerancia al estrés y en la clase 2 respuestas de afrontamiento ${ }^{(9)}$.

El diagnóstico enfermero Ansiedad (00146), se define como "sensación vaga e intranquilizadora de malestar o amenaza acompañada de una respuesta autonómica (el origen de la cual con frecuencia es inespecífico o desconocido para el individuo); sentimiento de aprensión causado por la anticipación de un peligro. Es una señal de alerta que advierte de un peligro inminente y permite al individuo tomar medidas para afrontar la amenaza"(10).

El manejo del paciente con trastorno de ansiedad en Atención Primaria resulta complejo, sobre todo, si consideramos la dificultad del diagnóstico diferencial, la necesidad de una terapéutica específica y la derivación a Atención Especializada cuando la etiología, el trastorno o las manifestaciones de la enfermedad así lo requieran ${ }^{(11)}$.

En cuanto al abordaje terapéutico de la ansiedad desde la disciplina enfermera, resulta importante la contribución que Peplau hace de la ansiedad y su tratamiento ${ }^{(12)}$. En el marco de este modelo conceptual, la autora menciona la ansiedad como una de las cuatro experiencias psicobiológicas que describe para identificar y explicar las acciones derivadas de experiencias que impulsan respuestas destructivas 0 constructivas por parte de las enfermeras y los pacientes. Este conocimiento proporciona una base tanto para la fijación de metas como para las intervenciones de enfermería ${ }^{(13)}$.

En este sentido, las técnicas de relajación y la educación sanitaria como intervenciones enfermeras en un contexto de asistencia grupal, se han mostrado efectivas para ayudar a los pacientes a controlar y/o disminuir los síntomas de ansiedad $^{(14-18)}$. Se han propuesto planes de cuidados enfermeros para la persona con ansiedad-estrés que utilizan entre otras estrategias de afrontamiento, la respiración controlada, la tensión y relajación muscular ${ }^{(19)}$. Diversos estudios evidencian que el uso de las intervenciones enfermeras como enseñanza grupo (5604), terapia de relajación simple (6040), educación sanitaria (5510) y disminución de la ansiedad $(5820)^{(14,16,18,20)}$ permiten abordar la respuesta de ansiedad hacia un afrontamiento más funcional de la misma con disminución del malestar y de la frecuentación de las consultas de atención primaria. Un estudio donde se utiliza la educación grupal en mujeres con diagnóstico enfermero ansiedad, demostró efectos beneficiosos en cuanto a mejoría en el grado de ansiedad y en la adquisición de habilidades para un adecuado manejo de la ansiedad ${ }^{(16)}$. Existen algunas Guías de Práctica Clínica que entre sus criterios de resultado destacan autocontrol de la ansiedad como parte de sus planes de cuidados para brindar una atención de calidad a los pacientes con esta problemática y ayudar al manejo de la ansiedad ${ }^{(21,22)}$.

En nuestra práctica clínica en salud mental, la demanda de ayuda por parte de las mujeres con diagnóstico enfermero de ansiedad va en aumento. Sin embargo no se han aportado suficientes evidencias respecto a la utilización de las intervenciones enfermeras (NIC) en estas mujeres y su evaluación atendiendo a los criterios de resultado enfermero (NOC).

Por todo lo anteriormente expuesto, consideramos conveniente elaborar una investigación en un ambiente comunitario de salud mental, con el propósito de evaluar la mejoría en el estado de las mujeres con diagnóstico enfermero ansiedad tras la 
implementación de las intervenciones enfermeras mediante el resultado NOC autocontrol de la ansiedad.

\section{MATERIAL Y MÉTODO}

\section{Diseño}

Estudio cuantitativo pre-experimental del tipo pretest-postest de un solo grupo.

\section{Población y ámbito de estudio}

La muestra fueron mujeres que presentaban el diagnóstico enfermero ansiedad. El periodo de estudio fue desde el 1 de mayo hasta el 30 de octubre de 2014.

La investigación se llevó a cabo en el Centro de Salud Mental (CSM) de Águilas, previa autorización por el Comité de Calidad del Hospital de referencia.

Se invitó a las participantes a formar parte del grupo, tras la obtención del consentimiento informado. Estas fueron reclutadas desde un listado de derivación por sus terapeutas de referencia.

La muestra estuvo compuesta por un grupo de 12 mujeres. Siguiendo las recomendaciones de la Clasificación de Intervenciones de Enfermería, para intervenciones enfermeras como terapia de grupo y grupo de apoyo, en las que se muestra que el tamaño óptimo de un grupo, no debe superar las 12 personas.

\section{Criterios de inclusión y exclusión}

Se incluyeron a mujeres adultas con edad comprendida entre los 18 y 65 años que presentasen diagnóstico enfermero ansiedad según taxonomía NANDA-I, y la voluntariedad en la participación en el estudio. Se excluyó a los sujetos varones, la presencia de patología mental grave (esquizofrenia, trastorno de personalidad, trastorno bipolar, depresión mayor y trastorno mental y del comportamiento debido a consumo de sustancias psicotropas) y el padecimiento de un trastorno mental derivado de enfermedad orgánica.

\section{Variables de estudio}

La variable independiente del estudio fue las intervenciones enfermeras en el área de la ansiedad a un grupo de mujeres con diagnóstico enfermero ansiedad. Se trató como variable dependiente las puntuaciones del criterio de resultado enfermero NOC autocontrol de la ansiedad.

\section{Intervenciones e instrumentos}

El grupo fue abordado por una Enfermera Especialista en Salud Mental y con experiencia en cuidados de ansiedad grupal e individual; a través del proceso de atención enfermero. Se realizó a cada paciente la valoración de enfermería por Patrones Funcionales de Salud (PFS) antes de recibir las intervenciones enfermeras $^{(23)}$, si cumplían con el criterio diagnóstico ansiedad según taxonomía NANDA-I ${ }^{(10)}$, eran incluidas para recibir las intervenciones enfermeras grupales en el área de la ansiedad. Al finalizar el grupo, se realizó una segunda valoración enfermera. Se programaron 8 sesiones grupales, a razón de 1 sesión a la semana de 1 hora de duración cada sesión. Al comenzar y al finalizar el grupo, se procedió a la cumplimentación de las puntuaciones de los indicadores del criterio de resultado autocontrol de la ansiedad; con la finalidad de identificar cambios en la respuesta de las pacientes. 
Las intervenciones enfermeras que se implementaron durante la fase de ejecución del proceso enfermero fueron a) disminución de la ansiedad (5820), terapia de relajación simple (6040), educación sanitaria (5510), enseñanza grupo (5604) y grupo de apoyo $(5430)^{(20)}$.

Los instrumentos empleados fueron los PFS adaptados en la práctica clínica en salud mental por Fornés ${ }^{(19)}$ y el criterio de resultado autocontrol de la ansiedad, recogido en la Clasificación de Resultados de Enfermería.

\section{Análisis estadístico}

Se utilizó la estadística descriptiva con el programa estadístico informático Statistical Package for Social Science (SPSS) versión 19.0. Para el análisis del criterio de resultado autocontrol de la ansiedad se han utilizado estadísticos de centralidad y de dispersión. Dado que el tamaño de la muestra es menor de 30 casos, se utiliza la prueba no paramétrica de los rangos con signo de Wilcoxon para conocer la existencia de diferencias entre dos muestras relacionadas.

\section{RESULTADOS}

Atendiendo a las variables sociodemográficas, el perfil tipo que refleja las características de la mayoría de las pacientes del estudio, se corresponde con una mujer casada que posee familia propia, con estudios primarios y una edad media de 39 años. En cuanto a la situación laboral, un 33\% trabaja y un 25\% presentan situación de desempleo.

El análisis del resultado NOC autocontrol de la ansiedad nos muestra diferencias significativas. En la tabla I mostramos los datos estadísticos del criterio de resultado enfermero, en el que se observan las diferencias estadísticamente significativas entre la medición basal y la medición después de la intervención, que representa los efectos de las intervenciones. Antes de la intervención, la media presentaba el valor de 22.5 y tras la misma de 37.8 , con una desviación típica de 3.22 y 7.11 respectivamente. La mediana antes y después de las intervenciones corresponde con los valores 21 y 37.5. El parámetro del test de los rangos de Wilcoxon presenta un valor de $\mathrm{Z}$ igual a 2.98 con una p igual a 0.003 .

Tabla I. Análisis estadístico del criterio de resultado 1402

\begin{tabular}{|l|l|l|l|l|l|l|l|l|}
\hline Variable & $\begin{array}{l}\text { Media } \\
\text { pre }\end{array}$ & $\begin{array}{l}\text { Desviación } \\
\text { típica pre }\end{array}$ & $\begin{array}{l}\text { Media } \\
\text { post }\end{array}$ & $\begin{array}{l}\text { Desviación } \\
\text { post }\end{array}$ & $\begin{array}{l}\text { Mediana } \\
\text { pre }\end{array}$ & $\begin{array}{l}\text { Mediana } \\
\text { post }\end{array}$ & Zw & $\mathbf{P}(\mathbf{0}-\mathbf{1})$ \\
\hline $\begin{array}{l}\text { Criterio de } \\
\text { Resultado } \\
\mathbf{1 4 0 2}\end{array}$ & & & & & & & & \\
$\begin{array}{l}\text { autocontrol } \\
\text { de la } \\
\text { ansiedad }\end{array}$ & 22.5 & 3.22 & 37.8 & 7.11 & 21 & 37.5 & -2.98 & 0.003 \\
\hline
\end{tabular}

Antes de intervenir, las pacientes presentaban una respuesta a su proceso de ansiedad, con aumento de la tensión, preocupación, insomnio, dificultades respiratorias, irritabilidad, dificultad para la concentración entre otras características definitorias del diagnóstico ansiedad. Tras la implementación de las intervenciones, obtenemos una mejoría en la repuesta de ansiedad, con un aumento en la puntuación de los indicadores del criterio de resultado autocontrol de la ansiedad. 
Los indicadores que quedaron modificados en la totalidad de las participantes por las intervenciones enfermeras fueron: a) monitoriza la intensidad de la ansiedad (140201), b) utiliza las técnicas de relajación para reducir la ansiedad (140207), c) refiere disminución de la duración de los episodios (140208), d) refiere ausencia de manifestaciones físicas de ansiedad (140215), e) ausencia de manifestaciones de una conducta de ansiedad (140216) y g) controla la respuesta de ansiedad (140217). Esto nos lleva a destacar los esfuerzos de adaptación realizados por las pacientes, con la finalidad de mejorar la capacidad en el afrontamiento de su respuesta de ansiedad (gráfico 1).

\section{Gráfico 1. Evolución de indicadores del NOC Autocontrol de la ansiedad}

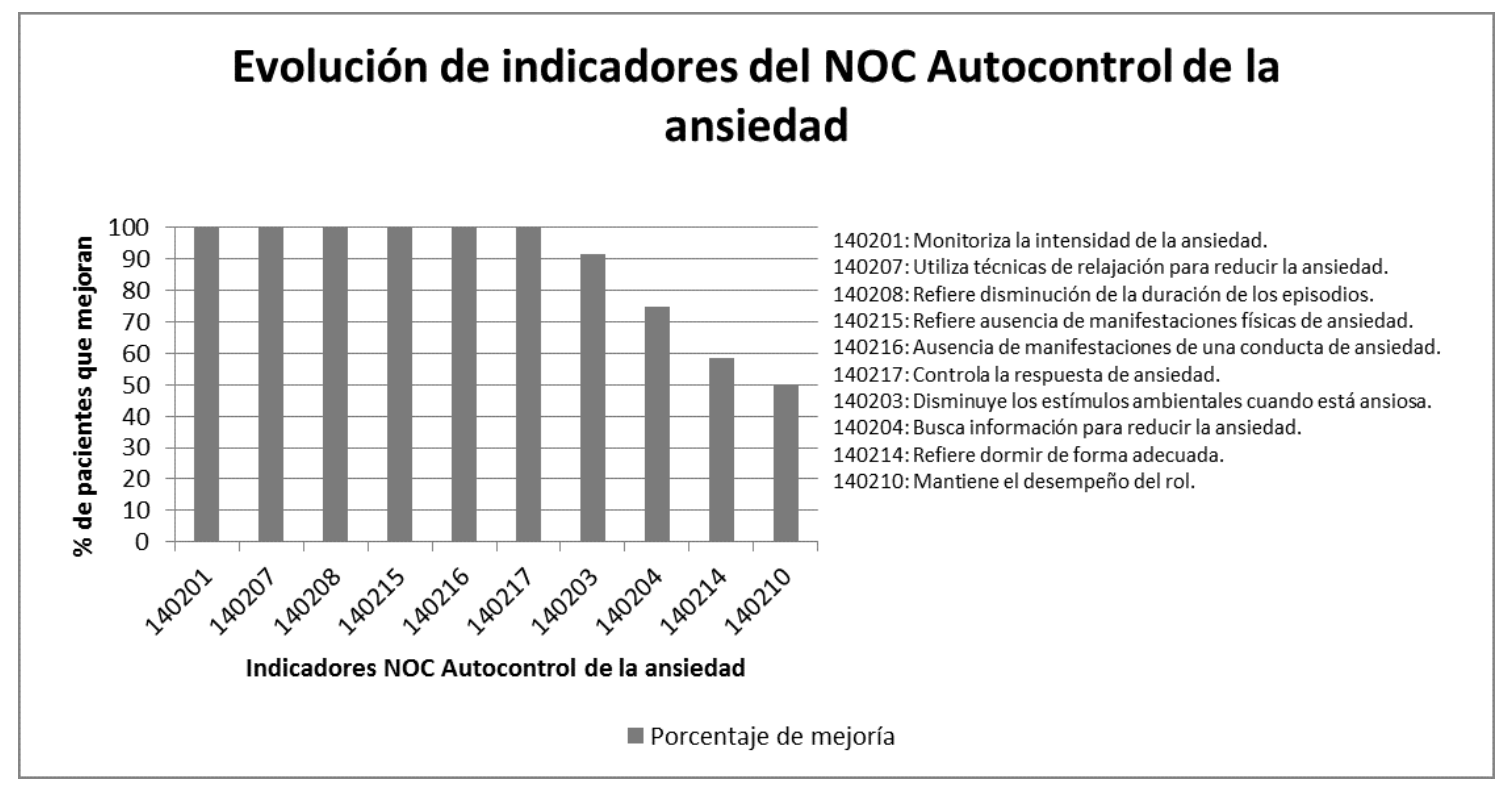

\section{DISCUSIÓN}

Basados en la literatura y en nuestra práctica clínica, planteamos la realización de intervenciones enfermeras grupales, con el fin de que las mujeres aprendieran a manejar su respuesta de ansiedad de manera más funcional. Destacamos la efectividad de los cuidados a través del cambio en los datos de valoración de los PFS, con especial mención al PFS adaptación tolerancia al estrés (P10). Si bien, en consonancia con el trabajo de algunos autores que atienden al carácter interdependiente de los PFS ${ }^{(24)}$, otros patrones también son influidos en el sentido de una mejoría clínica integral como el de actividad-ejercicio y autopercepciónautoconcepto.

La valoración de los patrones de salud refleja una mejoría de la respuesta de las pacientes con disminución de las dificultades respiratorias, de la tensión, preocupación, inquietud, irritabilidad así como de la duración de los episodios de ansiedad, entre otras características definitorias.

Las puntuaciones de los indicadores del criterio de resultado NOC obtenidas nos indican que las intervenciones enfermeras realizadas mejoran el estado de las mujeres con diagnóstico enfermero ansiedad. Las intervenciones terapia de relajación simple y disminución de la ansiedad de nuestro plan de cuidados coinciden con los 
trabajos de Ocio Aracama y Fornés ${ }^{(14,25)}$ utilizados para el control de la ansiedad. Las participantes han adquirido un procedimiento que pueden implementar cuando lo necesiten, con la finalidad de emitir una respuesta contraria a su estado de ansiedad conducente a resultados de salud positivos.

Otras intervenciones de enfermería como educación sanitaria, enseñanza grupo y grupo de apoyo han aportado beneficios en la adquisición de conocimientos y habilidades, que las mujeres han aplicado para el control del estado de ansiedad. Son algunos los estudios que desde un entorno de interacción grupal han mostrado resultados favorables encaminados a disminuir y controlar el estado de ansiedad $^{(18,26,27)}$, sin embargo, no son tantos los trabajos localizados, que han empleado estas intervenciones con mujeres, utilizando además los indicadores de criterios de resultado para evaluar la respuesta en salud. De ahí que entendemos la importancia de intervenir en Salud Mental Comunitaria desde un grupo, para mejorar el afrontamiento de la ansiedad, y en consonancia con las investigaciones de Roy, esta mejora se consigue cuando la enfermera ayuda al individuo a afrontar de manera efectiva los cambios derivados de un entorno continuamente cambiante ${ }^{(28)}$.

La intervención grupal ha sido objeto de interés en la aplicación de la relajación. En este sentido, un estudio realizado en un Centro de Salud Mental evalúa la efectividad de la relajación grupal en mujeres con trastornos por angustia y ansiedad generalizada. Los autores concluyen aseverando la efectividad del mismo en la disminución de las situaciones temporales de ansiedad y en menor grado la tendencia habitual a reaccionar de forma ansiosa ${ }^{(29)}$. Otro estudio, realizado en pacientes con ansiedad y depresión, muestra una disminución de los síntomas, al finalizar las intervenciones enfermeras grupales ${ }^{(18)}$.

Entendemos que pueden ser limitaciones del estudio, la ausencia de seguimiento de las mujeres participantes en los meses posteriores para conocer si se mantiene la mejoría conseguida y la carencia del grupo control. Además, el déficit de investigaciones enfermeras que estudien la ansiedad como diagnóstico enfermero desde una perspectiva de género en un contexto de salud mental y utilizando lenguajes enfermeros estandarizados, dificulta el marco de actualización de resultados en salud en esta área de conocimiento.

\section{CONCLUSIONES}

Entre las principales conclusiones del estudio cabe mencionar que la herramienta enfermera NOC nos ha permitido medir el estado de las pacientes a lo largo del proceso de cuidados y nos ha facilitado conocer la mejoría de la respuesta de las pacientes que reciben las intervenciones enfermeras.

El criterio de resultado NOC que vertebra la investigación autocontrol de la ansiedad nos permite valorar los cambios en la respuesta de ansiedad de las pacientes del estudio. En este sentido, las pacientes consiguieron un mejor control de la respuesta de ansiedad, mediante las intervenciones enfermeras realizadas. Pensamos que deben fomentarse los estudios de resultados en salud que aborden la problemática de los pacientes de salud mental, usando para ello metodología y lenguajes enfermeros. 


\section{REFERENCIAS}

1. Herrán A, Rodríguez Cabo B, Vázquez Barquero JL. Trastornos Depresivos. En: Vázquez Barquero JL, editor. Psiquiatr En Aten Primaria. $2^{\mathrm{a}}$. Madrid: Aula Médica; 2007. p. 223-49.

2. Alonso JT, Angermeyer MC, Bernert S, Bruffaerts R, Brugha T, Bryson H, et al. Prevalence of mental disorders in European: results from the European Study of the epidemiology of mental disorders (ESEMEeD) project. Acta Psychiatr Scand Suppl. 2004;(420):21-7.

3. Piqué Prado E. Mujer y salud desde una perspectiva de género. Metas Enferm. 2003;6(53):50-4.

4. Arenas M, Puigcerver A. Diferencias entre hombres y mujeres en los trastornos de ansiedad: una aproximación psicobiológica. Escr Psicol [Internet]. 2009 [citado 19 jun 2012];3(1):20-9. Disponible en: http://www.escritosdepsicologia.es/esp/numanteriores/vol3num1.html

5. Velasco Arias S, López Doriga B, Tourné García M, Calderó Bea MD, Barceló Barceló I, Luna Rodríguez C. Evaluación de una intervención biopsicosocial para el malestar de las mujeres en atención primaria. Feminismo/s. 2007;(10):111-31.

6. World Health Organization [Internet]. Geneva $(\mathrm{CH})$; Gender and women's mental health; 2013 [citado 4 may 2011]. Disponible en: http://www.who.int/mental_health/prevention/genderwomen/en

7. Maser JD, Patterson T. Spectrum and nosology: implications for DSM-V. Psychiatr Clin North Am. 2002;25(4):855-85.

8. Organización Mundial de la Salud (OMS). Clasificación de los Trastornos Mentales y del Comportamiento (CIE-10): Descripciones clínicas y pautas para el diagnóstico. $10^{\mathrm{a}}$. Madrid: Meditor; 1992.

9. Herdman TH, NANDA International, editores. NANDA International nursing diagnoses: definitions \& classification 2015 - 2017; ed. by T.Heather Hermann. 10. Aufl. Chichester: Wiley-Blackwell; 2014.

10. Heather Herdman T. NANDA International. Diagnósticos enfermeros: definiciones y clasificación 2009-2011. Madrid: Elsevier España; 2010.

11. Grupo de Trabajo de la Guía de Práctica Clínica para el Manejo de Pacientes con Trastornos de Ansiedad en Atención Primaria. Guía de Práctica Clínica para el manejo de Pacientes con Trastornos de Ansiedad en Atención Primaria [Internet]. Madrid: Unidad de Evaluación de Tecnologías Sanitarias. Agencia Laín Entralgo; 2008. Report No.: UETS $N^{\circ}$ 2006/10. Disponible en: http://www.guiasalud.es/GPC/GPC_430_Ansiedad_Lain_Entr_compl.pdf

12. Barkway P. Theories on mental health and illness. En: Ruth E, Evans K, Nizette D, editores. Psychiatr Ment Health Nurs. 2a . Australia: Elservier; 2009. p. 11933.

13. Carey ET, Rasmussen LN, Searcy B, Stark NL. Capítulo 16, Hildegard E. Peplau. Enfermería psicodinámica. En: Marriner A, editor. Model Teor Enferm. $1^{a}$. Barcelona: Ediciones Rol; 1989. p. 157-70.

14. Ocio Aracama C. Grupo de habilidades cognitivos-conductuales y de control de la respiración en el manejo de la ansiedad en Atención Primaria. Jalgui Col Of Enferm Gipuzkoa. 2010;(45):10-6.

15. Carretero Román, J. ¿Es más efectiva la relajación a nivel grupal que individual para disminuir la ansiedad en fobias específicas? Nure Inv. 2009;40.

16. Tierno Reina M, Cassinello Espinosa C, Rey Camacho E, Maldonado Tierno S. Educación grupal en mujeres con diagnóstico enfermero de ansiedad. Metas Enferm. 2003;6(52):6.

17. Mateos Rodilla J. La relajación. El arte de aflojar y soltar. Rev Rol Enferm. 2002;25(9):22-7. 
18. Casañas Sánchez R, Raya Tena A, Ibánez Pérez L, Valls Colomer MM. Intervención grupal psicoeducativa en pacientes con ansiedad y depresión en Atención Primaria de Barcelona. Aten Primaria. 2009;41(4):227-8.

19. Fornés Vives J, editor. Plan de cuidados para la persona con ansiedad-estrés. Enferm Salud Ment Psiquiátrica Planes Cuid. Madrid: Panamericana; 2006. p. 37-49.

20. Bulecheck GM, Butcher HK, Dochterman JM. Clasificación de Intervenciones de Enfermería (NIC). $5^{\text {a }}$. Barcelona: Elsevier España; 2009.

21. Ariz Larumbe ME, Cariñena Lezaun A, García Ruiz MA, Moros Borgoñón MA, Pascual Pascual P, Salaberri Nieto A. Manejo de la ansiedad en Atención Primaria: Guía de actuación. Servicio Navarro de Salud; 2009.

22. Planes de cuidados Enfermeros en SM. Cuaderno Técnico de Salud Mental. Servicio Extremeño de Salud; 2008.

23. Alfaro R. Aplicación del proceso de enfermería: guía práctica. Barcelona: Doyma; 1992.

24. Fornés Vives J, Carballal Balsa MC. Enfermería de salud mental y psiquiátrica: guía práctica de valoración y estrategias de intervención. Madrid: Médica Panamericana; 2001.

25. Fornés Vives J. Enfermería de salud mental y psiquiátrica: planes de cuidados. Madrid: Editorial Médica Panamericana; 2005.

26. Melgar Mairal M, Chorén Freire MJ, Campo Vázquez F, Díaz Ortiz MA, Campo Vázqued C, Cascón García M. Terapia grupal en síndrome ansioso depresivo y situaciones de estrés. Metas Enferm. 2005;8(8):20-3.

27. Soriano González J. Estudio cuasi-experimental sobre las terapias de relajación en pacientes con ansiedad. Enferm Global [Internet]. 2012;11(2):39-53. Disponisble en: http://revistas.um.es/eglobal/article/view/138971

28. Blue CL, Brubaker KM, Papazian KR, Riester CM. Capítulo 25, Callista Roy. Modelo de adaptación. En: Marriner A, editor. Model Teor Enferm. Barcelona: Rol; 1989. p. 260-74.

29. Tello Bernabé ME, Téllez Arévalo A, Ruiz Serrano A, De Frutos Martín MA, Alfaro $R$. Técnicas grupales y relajación en el tratamiento de algunos subtipos de ansiedad: un estudio de intervención controlado no aleatorio. Aten Primaria. 1997;2(19):67-71.

Recibido: 29 de mayo 2016;

Aceptado: 23 de agosto 2016

ISSN 1695-6141

(c) COPYRIGHT Servicio de Publicaciones - Universidad de Murcia 